\title{
Temperature influence compensation in microbolometer detector for image quality enhancement
}

\author{
by M. Krupiński*, T. Sosnowski*, H. Madura* and S. Gogler*
}

\begin{abstract}
* Military University of Technology, 00-908, Gen. Sylwestra Kaliskiego Str., Warsaw, Poland, michal.krupinski@wat.edu.pl, tomasz.sosnowski@wat.edu.pl, henryk.madura@wat.edu.pl, slawomir.gogler@wat.edu.pl
\end{abstract}

\begin{abstract}
In the article a non-uniformity correction method is presented which allows to compensate for the influence of detector's temperature drift. Presented method utilizes estimated dependency between output signal of detectors and their temperature. In the presented method, the dependency between output signal value and the temperature of the detector is estimated during time of starting detector. The coefficients are estimated for every pixel. Proposed method allows to compensate the influence of detectors temperature fluctuation and increase a time between shutter actuation process. Additional lenses coefficients allow to compensate ambient temperature fluctuation.
\end{abstract}

\section{Introduction}

The temperature of a detector array has important impact on a value of output signal and its parameters like NETD $[1,2,4]$. Therefore even insignificant change of detector temperature has a crucial influence on quality of nonuniformity correction (NUC) process, which has an affects on image quality.

Figure 1a represents an image after two point correction for temperature of array detector equal to $29^{\circ} \mathrm{C}$. Detector temperature change of $3^{\circ} \mathrm{C}$ causes deterioration of non-uniformity correction process and as a result four times larger fixed pattern noise (FPN) (figure $1 \mathrm{~b}$ ).

Because of significant impact of a microbolometer array temperature on infrared image quality, it's necessary to compensate for the influence of the temperature on non-uniformity correction process. In the most common applications two approaches are used. The first of them is microbolometer array temperature stabilization by thermoelectric cooler with a special controller. The second is updating offset coefficients by using a shutter. Sometimes they are used together. Both of them have disadvantages. The first case needs considerable amount of energy. The second one needs reference target and some mechanical procedure to place the target at the front of the detector $[3,6]$. Additionally during calibration the reference target (shutter) blocks the radiation from the scene interrupting measurements with such thermal camera. In military application interrupted observation have critical impact during combat operations. In this case it is important to increase the time between shutter actuation process by compensating image deterioration caused by temperature drift.

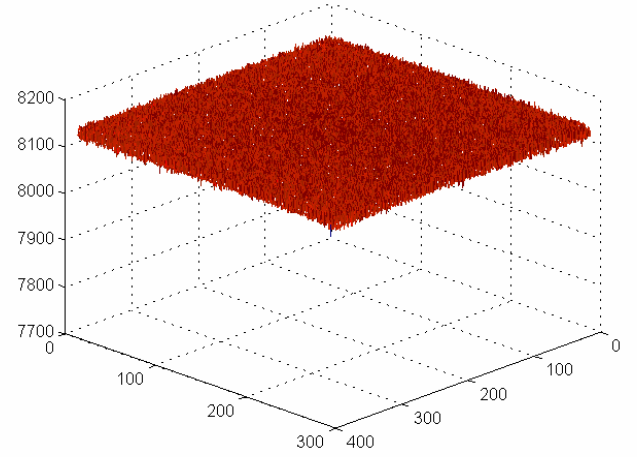

a)

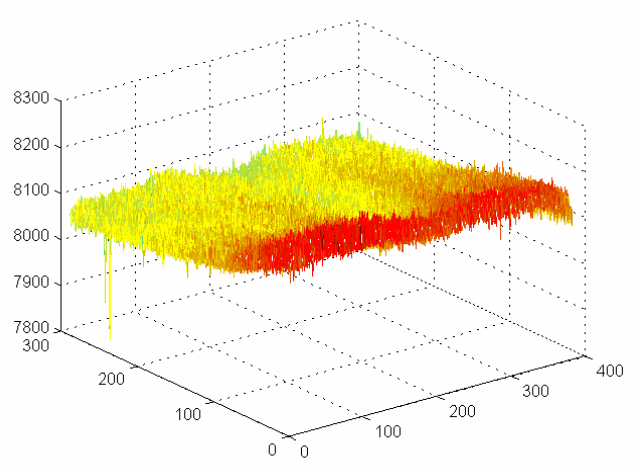

b)

Fig. 1. Detector array signal after NUC at detector temperature equal to $T=29^{\circ} \mathrm{C}$ (a), and after temperature drift of detector by $3^{\circ} \mathrm{C}$ with the same NUC coefficients

In typical solution the NUC coefficients are calculated for a specified temperature of a detector. In the case of temperature change of the detector the OFFSET coefficients must be recalculated to improve image quality. Therefore it 


\subsection{1/qirt.2016.068}

is very important to find out what is the relationship between the NUC coefficients and non-uniformity of a corrected image during temperature changes of the detector. Impact of the detector array temperature on correction quality were specified on the basis of registration prepared at a laboratory test bench with an infrared camera and a black body [7]. Temperature of the microbolometer detector was set and stabilized by a thermoelectric cooler. Registration was performed for three temperatures of the black body and four temperatures of the infrared detector. It allowed to estimate changes in Residual Non uniformity (RNU) (1) as a function of the detector array temperature.

$$
\begin{array}{r}
R N U=\frac{S_{x}}{\bar{X}} \cdot 100 \%, \\
S_{x}=\sqrt{\frac{1}{M N} \sum_{i=1}^{M} \sum_{j=1}^{N}\left[\bar{X}-X_{i j}\right]^{2}},
\end{array}
$$

where $\mathrm{Sx}$ - standard deviation of the image defined by equation (2), $\bar{X}$ - mean value of the image, $\mathrm{x}_{\mathrm{ij}}$ - value of output signal for bolometer i,j.

The previous research [7] shows that differences between following value of the RNU are rather inconsistent, for a constant GAIN table. Additionally value of calibration points used to calculate GAIN coefficients has bigger impact on the RNU value than a temperature drift of detector. The same test was performed for the OFFSET table coefficients. Previous work has showed that changes in detector temperature have much bigger influence on the RNU when the OFFSET coefficient are constant. This result allow to conclude that temperature of detector has more significant impact on the OFFSET coefficients than a GAIN.

In the proposed method a compensation of influence of microbolometer array temperature on image nonuniformity, bases on calculation of additional coefficients which allows to compensate for signal error caused by temperature drift. The characteristic of output signal as a function of detector temperature is presented at figure $2 \mathrm{~b}$. It is assumed that the OFFSET changes of the output signal as a function of detector temperature can be approximated by non-linear function in the range of $\pm 5^{\circ} \mathrm{C}$ around detector temperature measured after start of the camera. The characteristic of the output signal as a function of time during start of the camera is presented at figure $2 a$.

\section{Non-uniformity correction with temperature drift compensation}

In the proposed method one needs to determine three coefficients tables: GAIN, drift compensation coefficients (DCC) of the detector array and lens coefficient (LC). The GAIN and LC coefficients are determined at a test stand and are stored in the camera memory. Only the DCC are calculated in the camera.

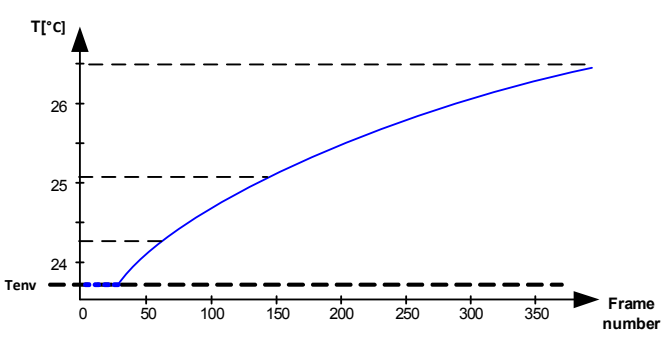

a

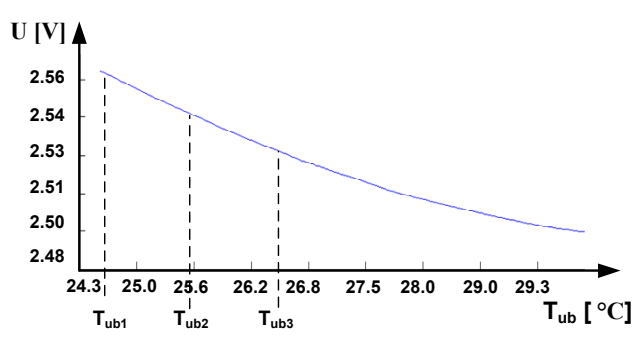

b

Fig. 2 Increase of FPA temperature during start (a) and detectors signal dependency of detector array temperature (b)

The DCC coefficients are calculated at base of OFFSET tables (3). DCC is non-linear approximation of OFFSET signal as a function of temperature of the detector array (Fig. 2b). OFFSET is calculated base on radiation flux coming from reference body, called shutter, installed in the camera, temporarily blocking radiation from the scene [6]. The OFFSET tables are determined at three different temperatures of the detector during natural heating up of the detector after switching the camera on. Power consumption of the bolometer array and a readout circuit cause natural heating up of the detector [5]. DDC is a second order polynomial coefficients to approximate OFFSET change with 


\subsection{1/qirt.2016.068}

temperature of the detector. Compensation of influence of the temperature drift on output signal is described by formula (3):

$$
\widetilde{U}_{i j}(\Phi)=U_{i j}^{*}(\Phi)+\mathbf{D C} \mathbf{C}_{\mathbf{i j}, \mathbf{x}} \cdot \mathbf{T}_{\mathbf{u b}, \mathbf{x}}
$$

where: $\widetilde{U}_{i j}(\Phi)$ - output signal of bolometer $i, j$ after compensation, $U_{i j}^{*}(\Phi)$ - value of output signal before compensation, $\mathrm{T}_{\mathrm{ub}}$ - actual detector array temperature, $D C C_{i j, x}$ - compensation coefficient of second order approximation for signal error as a function of detector temperature

where:

$$
\begin{aligned}
\mathbf{D C C}_{\mathbf{i j}} & =\mathbf{I}_{\text {errTub }} \cdot \mathbf{T}_{\mathbf{u b}}^{-1} \\
I_{e r r T u b} & =\bar{U}_{i j}\left(T_{u b}\right)-U_{i j}\left(T_{u b}\right),
\end{aligned}
$$

$U_{i j}\left(T_{u b}\right), \bar{U}_{i j}\left(T_{u b}\right)$ - response of detector i,j on constant reference radiation for temperatures set of detector array at $\mathrm{T}_{\mathrm{ub}}$ and mean value for all detectors, $I_{\text {errTub }}$ - offset signal for a specific temperature of the detector.

The equations (3) takes under consideration only temperature of the detector. But in fact the changes of environment temperature effects the temperature of the detector and temperature of the lens. In this case detector is exposed to radiation coming from observed scene (or shutter during calibration), and lens [6]. The research presented in the article [3] shows that ones have to determine the factors for compensating influence of the lens temperature change on the detector output signal. The addition of the lens coefficients allows to separate a phenomenon of detector temperature influence from the lens (camera housing) influence. The figure 3a shows a case where only the detector temperature is changed. Such conditions can be achieved by a thermoelectric cooler attached to the detector or by recording the output signal within a few minutes after the start of the camera. Under normal operation, the lens and detector temperature varies with the ambient temperature (figure $3 \mathrm{~b}$ ).

The effect of the environment temperature change can be reduced by calculating table coefficient which allows to determine signal error caused by temperature change of the lens (environment). Procedure of determination of "lens coefficient" LC consists of registration two images where each of them represent radiation of different lens' temperatures $\left(T_{\text {case } 1}=24, T_{\text {case } 2}=32\right)$. The black body temperature $T_{b b}$ and microbolometer temperatures $T_{u b}$ must be constant. The procedure of calculation is represented by formula (6) :

$$
L C_{i j}=\frac{\breve{U}_{i j}\left(T_{\text {case } 2}\right)-\breve{U}_{i j}\left(T_{\text {case } 1}\right)}{T_{\text {case } 2}-T_{\text {case } 1}}
$$

,where $\breve{U}_{i j}\left(T_{\text {case } 1}\right)$, and $\breve{U}_{i j}\left(T_{\text {case } 2}\right)$ :

$$
\begin{aligned}
& \breve{U}_{i j}\left(T_{\text {case } 2}\right)=\bar{U}\left(T_{\text {case } 2}\right)-U_{i j}\left(T_{\text {case } 2}\right) \\
& \breve{U}_{i j}\left(T_{\text {case } 1}\right)=\bar{U}\left(T_{\text {case } 1}\right)-U_{i j}\left(T_{\text {case } 1}\right)
\end{aligned}
$$

$L C_{i j}$ - lens coefficient for each detector, $\breve{U}_{i j}\left(T_{\text {case } 2}\right)$ - signal error for temperature $T_{\text {case } 2}, \breve{U}_{i j}\left(T_{\text {case } 1}\right)$ signal error for temperature $T_{\text {case } 1}, \bar{U}\left(T_{\text {case }}\right) \quad$ - mean signal value.

Finally, the equation for compensating ambient temperature change is : 


$$
\widetilde{U}_{i j}(\Phi)=U_{i j}^{*}(\Phi)+\mathbf{D C C} \mathbf{C}_{\mathbf{i j}} \cdot \mathbf{T}_{\mathbf{u b}}+L C_{i j} \cdot T_{C A S E}
$$

(1)

Fig. 3. RNU value of image as a function of detector array temperature a) and ambient temperature b).

The influence of the cases temperature on the output signal of the detector can be measured in climatic chamber by changing environment temperature with constant temperature of the detector and a black body [3].

The temperature of the FPA should be stabilised by thermoelectric cooler. In this case the temperature of the lens is the one variable in the camera.

\section{Measurement results}

To prepare an experiment a test stand must fulfill several conditions:

- the temperature of the bolometer array must be stable during measurement,

- ambient temperature should be stable for LC coefficients calculation,

- black body must be stable and must have uniform temperature,

To meet the above conditions microbolometer detector with lens was places in the climatic chamber. The temperature of the detector was stabilised by thermoelectric cooler with controller. Prepared test stand is presented at figure 5.

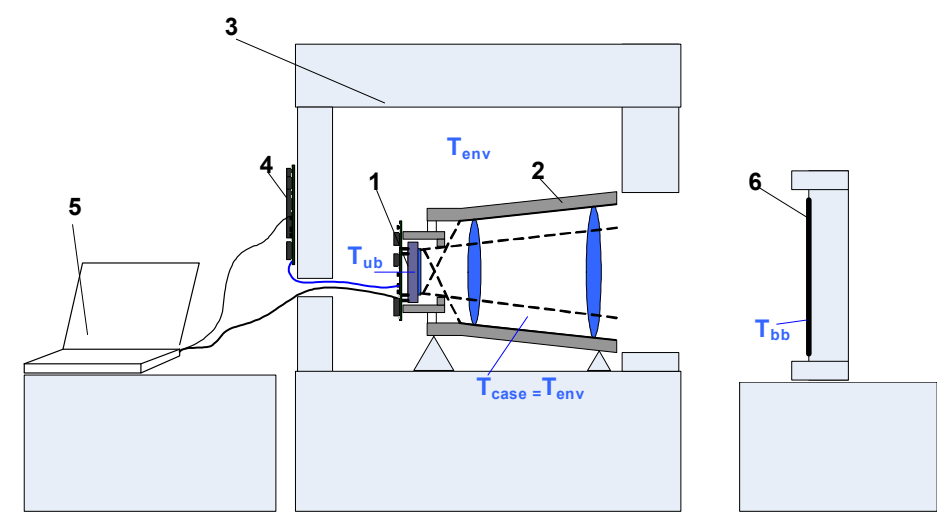

Fig. 4. Test stand for verifying proposed method

where:

1- micro bolometer with electronic readout circuit, 2- lens, 3- climatic chamber, 4- TEC driver, 5- computer with frame grabber, and control software, 6-black body, Tub- detector temperature, Tenv- environment temperature, Tcasecase (lens) temperature, $\mathrm{T}_{\mathrm{bb}}$ - black body temperature.

GAIN and OFFSET coefficients where calculated at the base of controlled black body. Tested detector allowed reading measurement data together with the temperature of detector array owing to temperature sensor inside. All images were captured through Orange-Tree evaluation board with special application designed by our team [4]. Quality of uniformity improvement by proposed algorithm was measured with use of RNU. Figure 5 compares images RNU after NUC correction to RNU after proposed correction method. It should be noted that the wider span of coefficient points we 


\subsection{1/qirt.2016.068}

get the wider temperature range compensation works. Additionally we can compare how RNU changes after involving environment temperature (Figure 5a).

Figure 5a shows how RNU changes after environment and micro bolometer temperatures drift. Environment temperature deteriorates corrections result. Involving lens coefficients can slightly expand temperature

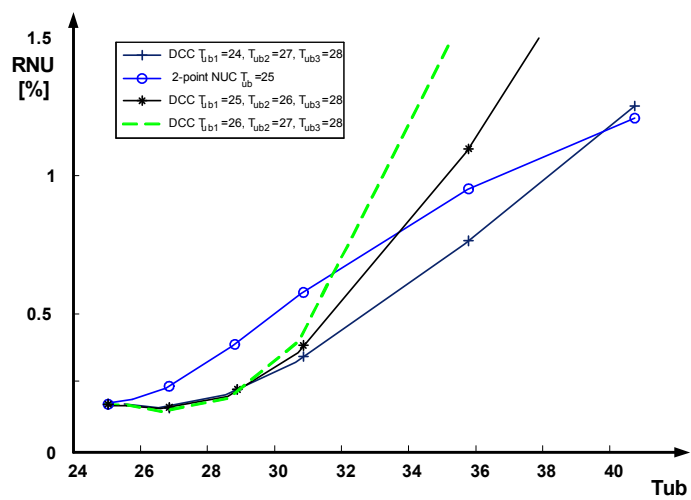

a

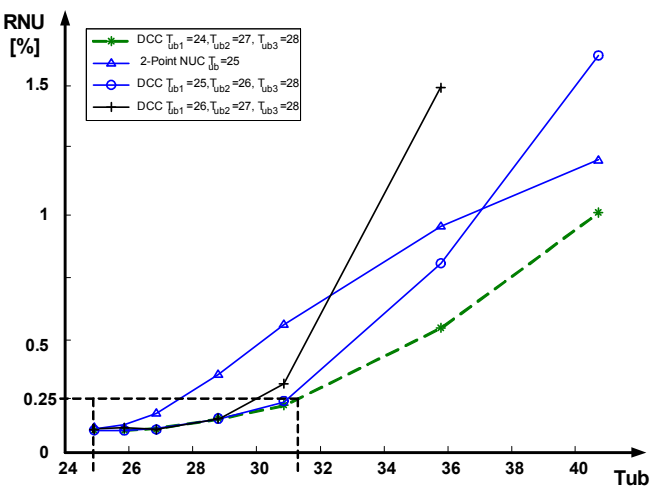

b

Fig. 5. RNU value of image as a function of detector array and environment temperature

range without needs of NUC actuation with shutter. Figure $5 \mathrm{~b}$ shows correction results after involving temperature influence compensation (DCC), and lens coefficients (LC). We can see that proposed method allows to maintain non uniformity of the images at lower level in wider environment temperature. It allows for longer use of camera without NUC actuation.

The compensation coefficient table was calculated for three ranges of the detector temperatures which allows to research its influence on compensation quality. The narrower the range of the detector's temperature, the smaller the value of the non-uniformity in compensated image. The result of proposed correction is presented at figure 6 for $4^{\circ} \mathrm{C}$ temperature drift.

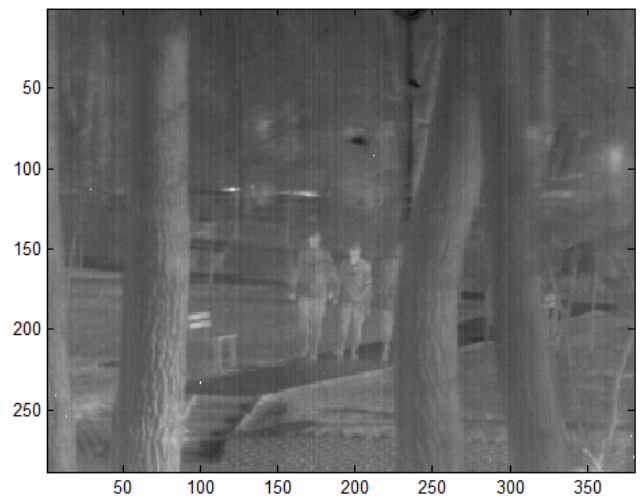

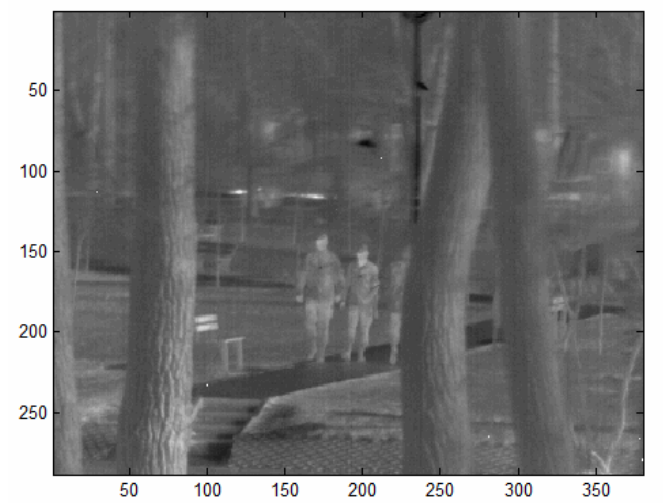

Fig. 6. Image after 2-point NUC (a) and DCC correction with LC compensation (b)

\section{CONCLUSION}

In the article a non-uniformity correction method is presented which allows to compensate for the influence of detector's temperature drift and ambient temperature fluctuation.

Presented method utilizes estimated dependency between output signal of detectors and their temperature during start of the camera. In the presented method, the shutter is used for establishing signal reference. Prepared measurements indicates that natural heating during start of the detector maybe used to establish compensation coefficients and increase time between shutter actuation. Additional LC coefficients calculated at the test stand improves non uniformity caused by lenses temperature change. Prepared measurement allow to conclude that additional lens coefficient increases time between shutter actuation. In this case a phenomenon of detector temperature influence is separated from lenses temperature change. 


\section{REFERENCES}

[1] Kruse, P. W., Uncooled Thermal Imagining, The Society of Photo+Optical Instrumentation Engineers 2002.

[2] Milton, A. F.; Barone, F. B.; Kruer, M. R. "Influence of nonuniformity on infrared focal plane array performance," Optical Engineering, Vol. 24, No. 5, pp. 855-862, 1985

[3] Krupiński, M., Bieszczad, G., Sosnowski, T., Gogler,S., Madura, H., "Non-uniformity correction with temperature influence compensation in microbolometer array," Proc. SPIE 9481, Image Sensing Technologies: Materials, Devices, Systems, and Applications II, 948113 May 13, 2015

[4] Sosnowski, T., Bieszczad, G., Madura, H., Kastek, M., Firmanty, K., "The calibration stand for thermal , camera module with cooled infrared focal plane array," Proceedings of SPIE, Vol. 7660, 2010, No. 76603Y

[5] Bieszczad, G., Kastek, M., "Measurement of thermal behavior of detector array surface with the use of microscopic thermal camera," MMS Volume 18, Issue 4, 2011, Pages 679-690

[6] Gogler, S., Bieszczad, G., Krupiński, M., Zarzycka, A., "A method of determination of voltage sensitivity of microbolometric focal plane array with optical system radiometric considerations," Przegląd Elektrotechniczny, Volume 89, Issue 10, 2013, Pages 65-68

[7] Krupiński, M., Bieszczad, G., Sosnowski, T., Madura, H., Gogler,S., "Non-uniformity correction in microbolometer array with temperature influence compensation” MMS, vol XXI No. 4, pp. 709 718, 2014r 